\title{
Fractal correlation property of heart rate variability in chronic obstructive pulmonary disease
}

This article was published in the following Dove Press journal:

International Journal of COPD

3I December 2010

Number of times this article has been viewed

\author{
Tatiana D Carvalho ${ }^{1,2}$ \\ Carlos Marcelo Pastre \\ Moacir Fernandes \\ de Godoy ${ }^{3}$ \\ Celso Fereira ${ }^{2}$ \\ Fábio $O$ Pitta $^{1,4}$ \\ Luiz Carlos de Abreu \\ Ercy Mara Cipulo Ramos \\ Vitor E Valenti2, \\ Luiz Carlos Marques \\ Vanderlei ${ }^{1}$ \\ 'Departamento de Fisioterapia \\ da Faculdade de Ciências e \\ Tecnologia, Universidade Estadual \\ Paulista, Presidente Prudente, São \\ Paulo, Brasil; '2Departamento de \\ Medicina, Disciplina de Cardiologia, \\ Universidade Federal de São Paulo, \\ São Paulo, Brasil; ${ }^{3}$ Departamento de \\ Cardiologia e Cirurgia Cardiovascular, \\ Faculdade de Medicina de São José \\ do Rio Preto, São José do Rio Preto, \\ São Paulo, Brasil; ${ }^{4}$ Laboratório de \\ Pesquisa em Fisioterapia Pulmonar, \\ Departamento de Fisioterapia, \\ Universidade Estadual de Londrina, \\ Londrina, Brasil; ${ }^{5}$ Departamento de \\ Morfologia e Fisiologia da Faculdade \\ de Medicina do ABC, Santo André, \\ Brasil
}

Correspondence: Tatiana Dias de Carvalho Departamento de Medicina,

Disciplina de Cardiologia, Universidade

Federal de São Paulo (UNIFESP), Rua

Napoleão de Barros, 715 - Térreo

04039-032, São Paulo, SP, Brasil

Tel +55 I | 55725462

Email carvalho.td।@gmail.com
Background: It was reported that autonomic nervous system function is altered in subjects with chronic obstructive pulmonary disease (COPD). We evaluated short- and long-term fractal exponents of heart rate variability (HRV) in COPD subjects.

Patients and methods: We analyzed data from 30 volunteers, who were divided into two groups according to spirometric values: $\operatorname{COPD}(n=15)$ and control $(n=15)$. For analysis of HRV indices, HRV was recorded beat by beat with the volunteers in the supine position for 30 minutes. We analyzed the linear indices in the time (SDNN [standard deviation of normal to normal] and RMSSD [root-mean square of differences]) and frequency domains (low frequency [LF], high frequency [HF], and LF/HF), and the short- and long-term fractal exponents were obtained by detrended fluctuation analysis. We considered $P<0.05$ to be a significant difference.

Results: COPD patients presented reduced levels of all linear exponents and decreased shortterm fractal exponent (alpha-1: $0.899 \pm 0.18$ versus $1.025 \pm 0.09, P=0.026$ ). There was no significant difference between COPD and control groups in alpha-2 and alpha-1/alpha-2 ratio.

Conclusion: COPD subjects present reduced short-term fractal correlation properties of HRV, which indicates that this index can be used for risk stratification, assessment of systemic disease manifestations, and therapeutic procedures to monitor those patients.

Keywords: pulmonary disease, chronic obstructive, heart rate, nervous system, cardiology

\section{Introduction}

Chronic obstructive pulmonary disease (COPD) is characterized by chronic airflow obstruction, which is not fully reversible, ${ }^{1,2}$ and significant systemic manifestations such as nutritional depletion, structural and functional changes of respiratory and peripheral muscles, and arrhythmias. ${ }^{3}$ Autonomic nervous system (ANS) dysfunction is also reported in COPD patients..$^{4-6}$ This may represent an important negative factor, as the ANS regulates internal functions of the body.

One method to evaluate the ANS function is the analysis of heart rate variability (HRV), the conventionally accepted term to describe the fluctuations in the intervals between consecutive heartbeats (RR intervals), which are indicated to influence the sinusal node. ${ }^{7}$ Previous studies indicate that COPD subjects present decreased HRV at rest compared with control subjects at the same age. ${ }^{8-10}$ Those studies regard specific changes of autonomic function in COPD-assessed HRV through linear methods in the time and frequency domains. However, to the best of our knowledge, no previous investigation has employed nonlinear methods to evaluate HRV in COPD patients. 
HRV analysis using nonlinear methods has been receiving attention. There is evidence that mechanisms involved in cardiovascular regulation likely interact between each other in a nonlinear fashion. ${ }^{11-13}$ One method used for this purpose is detrended fluctuation analysis (DFA), which quantifies the presence or absence of fractal correlation properties of the RR intervals. ${ }^{11}$ According to Tulppo et al ${ }^{14,15}$ fractal indices are able to detect slight changes in the dynamics of RR intervals better than conventional spectral analyses. Moreover, impairment of fractal correlation properties of short- and long-term dynamics of HRV helps clinical professionals to detect autonomic dysfunction and avoid disease development.

Based on the above considerations, we hypothesized that COPD patients would present altered heart rate dynamics. Therefore, this investigation was undertaken to evaluate shortand long-term fractal exponents of HRV in COPD subjects.

\section{Methods \\ Population}

We selected 15 patients ( 10 male) with a medical diagnosis of COPD, confirmed through spirometric test according to the Global Initiative for Chronic Obstructive Pulmonary Disease (GOLD), ${ }^{1}$ who were under treatment at the Centro de Estudos e Atendimento em Fisioterapia e Reabilitação da Faculdade de Ciências e Tecnologia, Universidade Estadual Paulista (Presidente Prudente, Brazil). When unhealthy conditions were reported, except for hypertension, the subject was not included in the sample. Furthermore, we excluded current smokers, individuals who were taking medication that influences the cardiac autonomic modulation, patients who presented COPD exacerbation in the last 2 months prior to the experimental protocol, and those who presented a restrictive pattern or no reproducible curves during the spirometric tests. The control group consisted of 15 healthy subjects ( 8 male), without diagnosis of COPD and who presented normal spirometric values. Group profiles are presented in Table 1.

All volunteers were informed about the procedures and objectives of the study, and on agreement they signed a consent letter. All work procedures were approved by the Ethics Committee in Research of our university (protocol number 246/08) and followed Resolution 196/96 of the National Health Council of October 10, 1996.

\section{Initial evaluation}

Before beginning the experimental procedure, we collected the following information: age, gender, height, weight, body
Table I Anthropometric and spirometric profile, baseline heart rate, and blood pressure of the COPD and control groups

\begin{tabular}{|c|c|c|c|}
\hline Variable & COPD $^{a}$ & Control $^{a}$ & $P$ \\
\hline Age (years) & $\begin{array}{l}73.93 \pm 6.61 \\
{[70.28-77.59]}\end{array}$ & $\begin{array}{l}68.73 \pm 7.27 \\
{[64.70-72.76]}\end{array}$ & 0.050 \\
\hline Weight (kg) & $\begin{array}{l}65.46 \pm 9.05 \\
{[60.45-70.47]}\end{array}$ & $\begin{array}{l}69.30 \pm 12.9 \\
{[62.13-76.4 I]}\end{array}$ & 0.358 \\
\hline Height (cm) & $\begin{array}{l}1.63 \pm 0.09 \\
{[1.51-1.75]}\end{array}$ & $\begin{array}{l}1.58 \pm 0.09 \\
{[1.44-1.74]}\end{array}$ & 0.112 \\
\hline BMI $\left(\mathrm{kg} / \mathrm{m}^{2}\right)$ & $\begin{array}{l}24.47 \pm 2.85 \\
{[22.89-26.05]}\end{array}$ & $\begin{array}{l}27.73 \pm 4.88 \\
{[25.03-30.43]}\end{array}$ & 0.036 \\
\hline FVC (\%) & $\begin{array}{l}81.59 \pm 18.60 \\
{[58.0-109.3]}\end{array}$ & $\begin{array}{l}97.18 \pm 14.62 \\
{[73.4-131.6]}\end{array}$ & 0.0164 \\
\hline $\mathrm{FEV}_{1}(\%)$ & $\begin{array}{l}51.69 \pm 16.32 \\
{[31-88]}\end{array}$ & $\begin{array}{l}97.18 \pm 14.62 \\
{[73.4-131.60]}\end{array}$ & $<0.000$ I \\
\hline $\mathrm{FEV}_{1} / \mathrm{FVC}$ & $\begin{array}{l}47.42 \pm 10.8(47.0) \\
{[41.43-53.40]}\end{array}$ & $\begin{array}{l}79.36 \pm 11.4(80.0) \\
{[73.06-85.66]}\end{array}$ & $<0.0001$ \\
\hline PEF (L/s) & $\begin{array}{l}3.36 \pm 1.5(3.53) \\
{[2.56-4.17]}\end{array}$ & $\begin{array}{l}6.38 \pm 2.16(6.17) \\
{[5.18-7.57]}\end{array}$ & 0.0003 \\
\hline HR (bpm) & $\begin{array}{l}70.73 \pm 10.9 \\
{[64.6-76.8]}\end{array}$ & $\begin{array}{l}65.93 \pm 7.6 \\
{[61.71-70.15]}\end{array}$ & 0.092 \\
\hline $\mathrm{SBP}(\mathrm{mm} \mathrm{Hg})$ & $\begin{array}{l}127.8 \pm 18.7(130) \\
{[90-160]}\end{array}$ & $\begin{array}{l}132.8 \pm 11.13(130) \\
{[118-160]}\end{array}$ & 0.5328 \\
\hline $\mathrm{DBP}(\mathrm{mm} \mathrm{Hg})$ & $\begin{array}{l}74.0 \pm 12.07(70) \\
{[58-100]}\end{array}$ & $\begin{array}{l}81.73 \pm 9.96(80) \\
{[76.2-87.2]}\end{array}$ & 0.0766 \\
\hline
\end{tabular}

Note: aMean \pm standard deviation (median) [confidence interval 95\%].

Abbreviations: COPD, chronic obstructive pulmonary disease; BMl, body mass index; FVC, forced vital capacity; $\mathrm{FEV}_{1}$, forced expiratory volume at the first second; PEF, peak expiratory flow; HR, heart rate; SBP, systolic blood pressure; DBP, diastolic blood pressure.

mass index (BMI), heart rate (HR), systolic blood pressure (SBP), and diastolic blood pressure (DBP). Anthropometric measurements were obtained following the standard recommendations proposed by Lohman et al. ${ }^{16} \mathrm{BMI}$ was calculated using the following formula: weight $(\mathrm{kg}) /$ height $\left(\mathrm{m}^{2}\right)$.

\section{Experimental protocol}

Data were collected under controlled temperature $\left(21^{\circ} \mathrm{C}-24^{\circ} \mathrm{C}\right)$ and humidity $(50 \%-60 \%)$, and volunteers were instructed to avoid consuming alcohol and caffeine for 24 hours before evaluation. Data were collected between $8 \mathrm{a} . \mathrm{m}$. and 11 a.m. in order to minimize the interference of circadian rhythm. All procedures necessary for the data collection were explained to the individuals, and the subjects were instructed to remain at rest and to avoid talking during the data collection.

After the initial evaluation the heart monitor strap was placed on each subject's thorax over the distal third of the sternum. The HR receiver (Polar S810i monitor, Polar Electro OY, Kempele, Finland) was placed on the wrist. This equipment has been previously validated for 
beat-by-beat measurements and for HRV analysis. ${ }^{17-20}$ The subjects were placed in the dorsal decubitus position on a cushion and remained at rest with spontaneous breathing for 30 minutes.

After the experimental procedures, we performed spirometric tests to identify and stratify the degree of bronchial obstruction. We used the Spirobank spirometer and a computer equipped with WinspiroPRO 1.1.6 software (Medical International Research, Rome, Italy). Data analysis was done according to the criteria described by the Guidelines for Pulmonary Function Tests. ${ }^{21}$

\section{Linear index of HRV evaluation}

HRV was recorded beat by beat through the monitoring process at a sampling rate of $1000 \mathrm{~Hz}$. During the period of higher signal stability, an interval of 5 minutes was selected, and series with more than $256 \mathrm{RR}$ intervals were used for analysis, ${ }^{7}$ following digital filtering complemented with manual filtering for the elimination of premature ectopic beats and artifacts. Only series with more than 95\% sinus rhythm were included in the study. ${ }^{13}$

To analyze HRV in the frequency domain, the low frequency (LF) (0.04-0.15 Hz) spectral component, which indicates both sympathetic and parasympathetic activity, 7,20 and high frequency (HF) $(0.15-0.40 \mathrm{~Hz})$ spectral component, which indicates parasympathetic activity, ${ }^{7,20}$ were used, ${ }^{2}$ as well as the ratio between these components (LF/HF). The spectral analysis was calculated using the Fast Fourier Transform algorithm. ${ }^{7,20}$ Analysis in the time domain was performed by means of SDNN (standard deviation of normalto-normal RR intervals) and RMSSD (root-mean square of differences between adjacent normal RR intervals in a time interval). For analysis of linear indexes in the time and frequency domains, we used the software HRV analysis. ${ }^{22}$

\section{Fractal analysis of HRV}

For the analysis of the fractal properties of the HR, DFA was applied to a time series of the RR intervals. This method is a modification of mean square root analysis of a random walk and is based on the analysis of fluctuations in the data following the removal of trends in the integrated time series, ${ }^{23}$ which allows the detection of intrinsic selfsimilarity embedded in the nonstationary time series. ${ }^{24} \mathrm{We}$ calculated the short-term fractal exponent (alpha-1), which corresponds to a period of 4-11 beats; long-term fractal exponent (alpha-2), which represents periods longer than 11 beats; ${ }^{25}$ and the alpha-1/alpha-2 ratio.
A software program available at PhysioNet (http:// www.physionet.org) - an online forum that fuses records of biomedical signals and software programs for the analysis of these signals - was used for the DFA. ${ }^{26}$

\section{Statistical analysis}

A Shapiro-Wilk test was used to evaluate the normality of the data. The nonpaired Student's $t$-test was applied to verify differences between parametric variables (age, weight, height, BMI, forced vital capacity [FVC], HR, SBP, DBP, HFms ${ }^{2}$, RMSSD, SDNN, alpha-1, alpha-2, and alpha-1/alpha-2). The Mann-Whitney test was used to verify differences between nonparametric variables $\left(\mathrm{LFms}^{2}, \mathrm{LF} / \mathrm{HF}\right.$, forced expiratory volume in the first second $\left[\mathrm{FEV}_{1}\right], \mathrm{FEV}_{1} / \mathrm{FVC}$, and peak expiratory flow $[\mathrm{PEF}]$ ). Differences were considered significant when the probability of a Type I error was lower than $5 \%(P<0.05)$.

The calculation of the study power (StatMate GraphPad Software version 2.00 for Windows, GraphPad Software, San Diego, CA, USA) with the number of subjects analyzed and significance level of 5\% (two-tailed test) confirmed a power higher than $80 \%$ to detect differences between the variables.

\section{Results}

Table 1 presents anthropometric measurements, spirometric values, HR, SBP, and DBP at rest in COPD and control groups. We observed that BMI, FVC, $\mathrm{FEV}_{1}, \mathrm{FEV}_{1} / \mathrm{FVC}$, and PEF were significantly lower in COPD subjects compared with the control group. There was no significant difference between the groups regarding HR, SBP, and DBP at rest.

Table 2 shows the values of alpha-1, alpha-2, and the ratio alpha-1/alpha-2 in COPD and control groups. We noted that the COPD group presented lower values of alpha-1 compared with the control group. However, we did not report differences between the COPD and control groups with respect to alpha-2 and the ratio alpha-1/alpha-2 values.

Table 2 Values of alpha-I, alpha-2, and alpha-I/alpha-2 ratio in the COPD and control groups

\begin{tabular}{llll}
\hline Variable & COPD $^{\mathbf{a}}$ & Control $^{\mathrm{a}}$ & $\boldsymbol{P}$ \\
\hline Alpha-I & $0.9 \pm 0.18$ & $1.02 \pm 0.09$ & 0.026 \\
& {$[0.79-1.00]$} & {$[0.97-1.07]$} & \\
Alpha-2 & $0.92 \pm 0.11$ & $0.91 \pm 0.12$ & 0.853 \\
& {$[0.86-0.98]$} & {$[0.85-0.98]$} & \\
Alpha-I/alpha-2 & $0.99 \pm 0.27$ & $1.13 \pm 0.13$ & 0.069 \\
& {$[0.84-1.14]$} & {$[1.05-1.20]$} & \\
\hline
\end{tabular}

Note: ${ }^{a}$ Mean \pm standard deviation [confidence interval 95\%].

Abbreviation: COPD, chronic obstructive pulmonary disease. 
Table 3 displays the values of the LF and HF indices in normalized units and $\mathrm{ms}^{2}$ as well as the LF/HF ratio in COPD and control groups. There was reduction of all indices in COPD patients compared with control subjects.

\section{Discussion}

The results of the present study suggest that there is a loss or disarrangement of the properties of short-term fractal correlations of HRV associated with a reduction in both sympathetic and parasympathetic activity in COPD subjects.

According to Tulppo et $\mathrm{al}^{15}$ and Acharya et al, ${ }^{24}$ in relation to exponents obtained by the DFA, values close to 1 are indicative of a fractal system, whereas values close to 0.5 are associated with random signal, where there is no correlation between the values. Therefore, our results indicate that COPD patients exhibit dynamic changes in HR toward a more dynamic random profile, which indicates a condition of loss of chaos.

Under conditions where the action of sympathetic and parasympathetic components of the ANS on HR is organized in a reciprocal behavior, ie, an increased activity of a system is accompanied by a decrease of another, there is a strong short-term fractal correlation expressed by increasing the value of alpha-1. ${ }^{14,27}$ Notwithstanding conditions in which there is increased activity of both ANS components (sympathetic and parasympathetic), loss of short-term HR fractal organization was noted, which decreased the value of alpha- $1 .^{15,28,29}$ According to our study, the analysis of linear indices in the time and frequency domains in $\mathrm{ms}^{2}$, as well as the ratio between these two components ( $\mathrm{LF} / \mathrm{HF}$ ), in COPD patients showed reduction of both parasympathetic and sympathetic components of the ANS compared with the control group. Furthermore, reduced activity of sympathetic and para-

Table 3 Linear index values of heart rate variability (frequency and time domains) in the COPD and control groups

\begin{tabular}{llll}
\hline Variable & COPD $^{\mathbf{a}}$ & Control $^{\mathrm{a}}$ & $\boldsymbol{P}$ \\
\hline LFms $^{2}$ & $28.80 \pm 24.48(20.0)$ & $|30.00 \pm 131.5|(88.0)$ & 0.0007 \\
& {$[15.24-42.36]$} & {$[57.17-202.83]$} & \\
HFms $^{2}$ & $18.93 \pm 9.89$ & $52.5 \pm 38.3$ & 0.005 \\
& {$[13.46-24.4 \mid]$} & {$[31.33-73.74]$} & \\
RMSSD & $11.23 \pm 3.48$ & $18.93 \pm 7.48$ & 0.002 \\
& {$[9.30-13.16]$} & {$[14.78-23.07]$} & \\
SDNN & $\mid 4.13 \pm 5.03$ & $25.4 \pm 9.5$ & 0.0004 \\
& {$[6-24]$} & {$[11-49]$} & \\
\hline
\end{tabular}

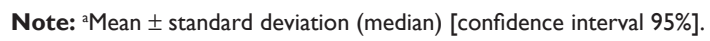

Abbreviations: COPD, chronic obstructive pulmonary disease; LF, low frequency; $\mathrm{HF}$, high frequency; ms, miliseconds; RMSSD, root-mean square of differences between adjacent normal RR intervals in a time interval; SDNN, standard deviation of normal-to-normal RR intervals. sympathetic components of the ANS in elderly patients with COPD compared with control subjects at similar age at rest and supine position was observed previously. ${ }^{4-6}$ Taken together, our findings and previous investigations suggest that the reduction of alpha-1 reflects a decrease in both ANS component activities.

The loss of short-term fractal correlation property that we found in COPD subjects is related to the occurrence of several adverse clinical events such as heart failure ${ }^{23,25}$ and acute myocardial infarction. ${ }^{30}$ In addition, reduction of alpha-1 was observed before spontaneous onset of atrial fibrillation in patients without structural heart disease ${ }^{27}$ associated with vulnerability to ventricular tachycardia ${ }^{23,30}$ and ventricular fibrillation. ${ }^{31}$ Exponent values lower than 0.85 were observed in patients with cardiovascular disease associated with increased mortality rate. ${ }^{28-30}$ These values are close to those found in our study (alpha- $1=0.899$ ) in COPD patients.

Our investigation suggests that COPD subjects present autonomic dysfunction, characterized by loss of short-term fractal correlation of HRV and reductions of sympathetic and parasympathetic activity. It corroborates the importance of the use of nonlinear dynamics analysis in the prognostic assessment of morbid states, due to its ability to evaluate the degree of loss of the patient's homeostatic behavior, considering the whole and not only the severity of the diseases alone.

Nonlinear methods of HRV analysis describe complex fluctuations in heart rhythm and are able to separate structures with nonlinear behavior in heartbeat time series more adequately than linear methods. ${ }^{12}$ They enable a better discrimination between individuals with normal and altered physiology, ${ }^{13}$ as well as a better understanding of the nature of the complex dynamic systems that occur in the human body in both health and sickness. ${ }^{32}$

In relation to long-term exponents (alpha-2) and alpha-1/ alpha-2 ratio, there was no difference between the COPD and control groups. Our findings suggest that COPD patients present autonomic dysfunction characterized by loss of short-term fractal correlation of HRV associated with sympathetic and parasympathetic activity reduction. Thus, interventions that may restore fractal dynamic properties of HR and increase HRV may have important clinical implications, and the performance of physical exercise programs should be emphasized. In a study that evaluated the effect of resistance training in healthy adults, Heffernan et al ${ }^{33}$ found improvement of fractal dynamic properties of HR, whereas Borghi-Silva et $\mathrm{l}^{4}$ investigated the effects of aerobic training 
on autonomic cardiac regulation in COPD subjects and also found changes reflected by improvement of HRV indices in the time and frequency domains.

Our research presents a couple of points that should be addressed. First, both groups presented hypertensive patients; however, basal HR, SBP, and DBP were not significantly different between the groups. Second, COPD subjects presented different degrees of obstruction. Nevertheless, Camillo et $\mathrm{al}^{5}$ have shown that COPD severity is not significantly related to HRV indices.

To the best of our knowledge, our findings are the first to evidence reduced alpha-1, the short-term fractal exponent, in COPD subjects. Our findings are relevant, as they indicate that these indices, obtained by a noninvasive and inexpensive method, may be useful both for clinical manifestations of systemic disease and for risk stratification and monitoring of therapeutic procedures performed with these patients.

In conclusion, COPD subjects present reduced shortterm fractal correlation of HRV, indicating sympathetic and parasympathetic activity reduction.

\section{Disclosure}

This study received financial support from Fundação para Desenvolvimento da Universidade Estadual Paulista (FUNDUNESP, process number 00704/08 - DFP).

\section{References}

1. Rabe KF, Hurd S, Anzueto A, et al; Global Initiative for Chronic Obstructive Lung Disease. Global strategy for the diagnosis, management, and prevention of chronic obstructive pulmonary disease: GOLD executive summary. Am J Respir Crit Care Med. 2007;176: $532-555$.

2. Seemungal TA, Hurst JR, Wedzicha JA. Exacerbation rate, health status and mortality in COPD - a review of potential interventions. Int J Chron Obstruct Pulmon Dis. 2009;4:203-223.

3. Dourado VZ, Tanni SE, Vale SA, et al. Systemic manifestations in chronic obstructive pulmonary disease. J Bras Pneumol. 2006;32:161-171.

4. Borghi-Silva A, Arena R, Castello V, et al. Aerobic exercise training improves autonomic nervous control in patients with COPD. Respir Med. 2009;103:1503-1510.

5. Camillo CA, Pitta F, Possani HV, et al. Heart rate variability and disease characteristics in patients with COPD. Lung. 2008;186:393-401.

6. Pantoni CBF, Reis MS, Martins LEB, Catai AM, Costa D, BorguiSilva A. Estudo da modulação autonômica da freqüência cardíaca em repouso de pacientes idosos com doença pulmonar obstrutiva crônica. Rev Bras Fisioter. 2007;11:35-41.

7. Task Force of the European Society of Cardiology and the North American Society of Pacing and Electrophysiology. Heart rate variability: standards of measurement, physiological interpretation and clinical use. Circulation. 1993;93:1043-1065.

8. Paschoal MA, Petrelluzzi KFS, Gonçalves NVO. Estudo da variabilidade da freqüência cardíaca em pacientes com doença pulmonar obstrutiva crônica. Rev Ciênc Med. 2002;11:27-37.

9. Pagani M, Lucini D, Pizzinelli P, et al. Effects of aging and of chronic obstructive pulmonary disease on RR interval variability. J Auton Nerv Syst. 1996;59:125-132.
10. Volterrani M, Scalvini S, Mazzuero G, et al. Decreased heart rate variability in patients with chronic obstructive pulmonary disease. Chest. 1996;106:1432-1437.

11. Huikuri HV, Makikallio TH, Perkiomaki J. Measurement of heart rate variability by methods based on nonlinear dynamics. J Electrocardiol. 2003;36:95-99.

12. Voss A, Kurths J, Kleiner HJ, et al. The application of methods of non-linear dynamics for the improved and predictive recognition of patients threatened by sudden cardiac death. Cardiovasc Res. 1996;31: 419-433.

13. Godoy MF, Takakura IT, Correa PR. Relevância da análise do comportamento dinâmico não-linear (Teoria do Caos) como elemento prognóstico de morbidade e mortalidade em pacientes submetidos à cirurgia de revascularização miocárdica. Arq Ciênc. 2005;12:167-171.

14. Tulppo MP, Kiviniemi AM, Hautala AJ, et al. Physiological background of the loss of fractal heart rate dynamics. Circulation. 2005;112: 314-319.

15. Tulppo MP, Hughson RL, Mäkikallio TH, Airaksinen KE, Seppänen T, Huikuri HV. Effects of exercise and passive head-up tilt on fractal and complexity properties of heart rate dynamics. Am J Physiol. 2001; 280:H1081-H1087.

16. Lohman TG, Roche AF, Martorell R. Anthropometric Standardization Reference Manual. Champaign: Human Kinetics Books; 1998.

17. Gamelin FX, Berthoin S, Bosquet L. Validity of the Polar S810 Heart Rate Monitor to measure R-R intervals at rest. Med Sci Sports Exerc. 2006;38:887-893.

18. Porto LGG, Junqueira Júnior LF. Comparison of time-domain short-term heart interval variability analysis using a wrist-worn heart rate monitor and the conventional electrocardiogram. PACE. 2008;32:43-51.

19. Vanderlei LC, Silva RA, Pastre CM, Azevedo FM, Godoy MF. Comparison of the Polar S810i monitor and the ECG for the analysis of heart rate variability in the time and frequency domains. Braz J Med Biol Res. 2008;41:854-859.

20. Vanderlei LC, Pastre CM, Hoshi RA, Carvalho TD, Godoy MF. Basic notions of heart rate variability and its clinical applicability. Rev Bras Cir Cardiovasc. 2009;24:205-217.

21. Pereira CAC, Neder JA. Diretrizes para testes de função pulmonar. J Pneumol. 2002;28(Suppl 3):S1-S238.

22. Niskanen JP, Tarvainen MP, Ranta-Aho PO, Karjalainen PA. Software for advanced HRV analysis. Comput Methods Programs Biomed. 2008; 76:73-81.

23. Peng CK, Havlin S, Stanley HE, Goldberger AL. Quantification of scaling exponents and crossover phenomena in nonstationary heartbeat time series. Chaos. 1995;5:82-87.

24. Acharya RU, Lim CM, Joseph P. Heart rate variability analysis using correlation dimension and detrended fluctuation analysis. ITBM-RBM. 2002;23:333-339.

25. Perkiömäki JS, Zareba W, Ruta J, et al; for IDEAL Investigators. Fractal and complexity measures of heart rate dynamics after acute myocardial infarction. Am J Cardiol. 2001;88:777-781.

26. Hautala AJ, Mäkikallio TH, Seppänen T, Huikuri HV, Tulppo MP. Shortterm correlation properties of RR interval dynamics at different exercise intensity levels. Clin Physiol Funct Imaging. 2003;23:215-223.

27. Hayano J, Takahashi H, Toriyama T, et al. Prognostic value of heart rate variability during long-term follow-up in chronic haemodialysis patients with end-stage renal disease. Nephrol Dial Transplant. 1999;14:1480-1488.

28. Vanderlei LC, Pastre CM, Júnior IF, de Godoy MF. Fractal correlation of heart rate variability in obese children. Auton Neurosc. 2010; 155:125-129.

29. Vanderlei LC, Pastre CM, Freitas Júnior IF, de Godoy MF. Analysis of cardiac autonomic modulation in obese and eutrophic children. Clinics. 2010;65:789-792.

30. Mäkikallio TH, Høiber S, Køber L, et al. Fractal analysis of heart rate dynamics as a predictor of mortality in patients with depressed left ventricular function after acute myocardial infarction. Am J Cardiol. 1999;83:836-839. 
31. Makikallio TH, Seppanen T, Airaksinen KE, et al. Dynamic analysis of heart rate may predict subsequent ventricular tachycardia after myocardial infarction. Am J Cardiol. 1997;80:779-783.

32. Higgins JP. Nonlinear systems in medicine. Yale J Biol Med. 2002; 75(5-6):247-260.
33. Heffernan KS, Sosnoff JJ, Fahs CA, Shinsako KK, Jae SY, Fernhall B. Fractal scaling properties of heart rate dynamics following resistance exercise training. J Appl Physiol. 2008;105:109-113.

\section{Publish your work in this journal}

The International Journal of COPD is an international, peer-reviewed journal of therapeutics and pharmacology focusing on concise rapid reporting of clinical studies and reviews in COPD. Special focus is given to the pathophysiological processes underlying the disease, intervention programs, patient focused education, and self management protocols.
This journal is indexed on PubMed Central, MedLine and CAS. The manuscript management system is completely online and includes a very quick and fair peer-review system, which is all easy to use. Visit $\mathrm{http} / / / \mathrm{www}$.dovepress.com/testimonials.php to read real quotes from published authors. 